\title{
Jacobi Manifolds, Contact Manifolds and Contactomorphism
}

\author{
Servais Cyr GATSE
}

Correspondence: Université Marien Ngouabi, Faculté des Sciences et Techniques BP: 69, Brazzaville, Republic of Congo

Received: March 29, 2021 Accepted: July 23, 2021 Online Published: July 29, 2021

doi:10.5539/jmr.v13n4p85 URL: https://doi.org/10.5539/jmr.v13n4p85

\begin{abstract}
Let $M$ be a smooth manifold and let $\mathscr{D}(M)$ be the module of first order differential operators on $M$. In this work, we give a link between Jacobi manifolds and Contact manifolds. We also generalize the notion of contactomorphism on $M$ and thus, we characterize the Contact diffeomorphisms.
\end{abstract}

Keywords: Jacobi manifolds, contact manifolds, differential operators, contactomorphism

\section{Introduction}

\subsection{Jacobi Manifolds}

The notion of Jacobi manifolds has been introduced by A. Lichnerowicz (Lichnerowicz, 1978). These manifolds are a generalization of the notion of locally conformally symplectic manifolds and of contact manifolds (which can be used in the representation of energy in thermodynamics). A Jacobi structure on a manifold $M$ is a pair $(\Lambda, E)$ where $\Lambda$ is a contravariant skew-symmetric 2-tensor and $E$ is a vector field on $M$ such that

$$
[\Lambda, \Lambda]=2 E \wedge \Lambda, \theta_{E} \Lambda=[E, \Lambda]=0
$$

with $\wedge$ the Grassmann wedge product and $\theta_{E}$ the Lie derivative with respect to the vector field $E$. The bracket defined in (1) is the Schouten-Nijenhuis bracket on multivectors (Koszul, 1985).

Using the classical Darboux's theorem, around every point of $M$ there exist canonical coordinates $\left(x_{1}, \ldots, x_{2 n}\right)$ and a local smooth function $f$ on $M$ such that

$$
E=e^{-f} \sum_{i=1}^{n}\left(\frac{\partial f}{\partial x_{i}} \frac{\partial}{\partial x_{n+i}}-\frac{\partial f}{\partial x_{n+i}} \frac{\partial}{\partial x_{i}}\right), \Lambda=e^{-f} \sum_{i=1}^{n} \frac{\partial}{\partial x_{n+i}} \wedge \frac{\partial}{\partial x_{i}}
$$

Defining a Jacobi structure on $(\Lambda, E)$ is equivalent to defining an internal composition law $\{\cdot, \cdot\}$ on $\mathscr{C}^{\infty}(M)$ such that for all $f, g \in \mathscr{C}^{\infty}(M)$

$$
\{f, g\}=\Lambda(d f, d g)+f \cdot E(g)-g \cdot E(f)
$$

where $d$ is the exterior differentiation operator (Okassa, 2007). The bracket $\{\cdot, \cdot\}$ is skew-symmetric and satisfies the Jacobi identity if and only if (1) is fulfilled. Furthermore, one has the locality condition

$$
\operatorname{supp}\{f, g\} \subseteq \operatorname{supp}(f) \cap \operatorname{supp}(g) .
$$

A natural generalization of Poisson bracket is Jacobi bracket. The only difference is that we only replace the Leibniz rule by

$$
\{f, g \cdot h\}=\{f, g\} \cdot h+g \cdot\{f, h\}-g \cdot h \cdot\{f, 1\}
$$

for all $f, g, h, 1 \in \mathscr{C}^{\infty}(M)$, where 1 is the unit of $\mathscr{C}^{\infty}(M)$.

Proposition 1. The Jacobi bracket $\{\cdot, \cdot\}$ defines a Lie algebra structure on $\mathscr{C}^{\infty}(M)$, if and only if (1) is satisfied.

The vector field $E$ is the hamiltonian vector field associated with the constant function 1 . We have $E(f)=\{1, f\}$, whence the Jacobi identity for $(1, f, g)$ yields

$$
E(\{f, g\})=\{E(f), g\}+\{f, E(g)\}
$$

which equivalent to $\theta_{E} \Lambda=0$. If the vector field $E$ vanishes, we recover the Poisson bracket.

Remark 2. (Lichnerowicz, 1977) Let $(\Lambda, E)$ be a Jacobi structure on a manifold $M$ and consider a product manifold $M \times \mathbb{R}$, the bivector $\widetilde{\Lambda}$ given by $\widetilde{\Lambda}=e^{-t}\left(\Lambda+\frac{\partial}{\partial t} \wedge E\right)$, where $t$ is the usual coordinate on $\mathbb{R}$. Then, $\widetilde{\Lambda}$ defines a Poisson structure on $M \times \mathbb{R}$. The manifold $M \times \mathbb{R}$ with the structure $\tilde{\Lambda}$ is called the poissonization of the Jacobi manifold $(M, \Lambda, E)$.

Note that a smooth manifold $M$ is a Jacobi manifold when the algebra of numerical functions of class $\mathscr{C}^{\infty}$ on $M, \mathscr{C}^{\infty}(M)$ admits a Jacobi algebra structure (Okassa, 2007). 


\subsection{Contact Manifolds}

By a contact manifold in this paper, we mean a $\mathscr{C}^{\infty}$ manifold $M^{2 n+1}$ together with a 1 -form $\alpha$ such that $\alpha \wedge(d \alpha)^{n} \neq 0$. In particular, $\alpha \wedge(d \alpha)^{n}$ is a volume element on $M^{2 n+1}$, so that a contact manifold is orientable. Also $(d \alpha)^{n}$ has rank $2 n$ on the Grassmann algebra $\Lambda T_{p}^{\star} M$ at each point $p \in M$, and thus we have a 1-dimensional subspace,

$$
\left\{X \in T_{p} M / d \alpha\left(X, T_{p} M\right)=0\right\}
$$

on which $\alpha \neq 0$ and which is complementary to the subspace defined by $\alpha=0$.

Therefore choosing $R_{p}$ in this subspace normalized by $\alpha\left(R_{p}\right)=1$, we have a global vector field $R$ satisfying $\alpha(R)=1$ and $d \alpha(R, X)=0$. In this case, the $\mathscr{C}^{\infty}(M)$-module of vector fields $\mathfrak{X}(M)$ admits a inner sum direct decomposition

$$
\mathfrak{X}(M)=\operatorname{Ker} \alpha \oplus \mathscr{C}^{\infty}(M) \cdot R,
$$

where, $R$ is called Reeb vector field of the contact structure $\alpha$. We deduce that $\theta_{R} \alpha=0$ and $\theta_{R} d \alpha=0$.

One denotes by $\mathscr{D}$ the contact distribution defined by the space

$$
\mathscr{D}_{p}=\left\{X \in T_{p} M / \alpha(X)=0\right\}=\operatorname{ker}(\alpha) .
$$

Roughly speaking, the meaning of the contact condition, $\alpha \wedge(d \alpha)^{n} \neq 0$, is that the contact subbundle is as far from being integrable as possible.

By a smooth manifold, we will mean a paracompact connected finite dimensional $\mathscr{C}^{\infty}$ manifold without boundary.

Here one lists a few examples of contact manifolds and Reeb vector field.

(i) The pair $\left(\mathbb{R}^{2 n+1}, \alpha=d z+\sum_{i=1}^{n} x_{i} d y_{i}\right)$ is a contact manifold with Reeb vector field $\frac{\partial}{\partial z}$. Indeed, if $R=a \frac{\partial}{\partial z}+b_{i} \frac{\partial}{\partial x_{i}}+c_{i} \frac{\partial}{\partial y_{i}}$, then $1=\alpha(R)=a-b_{i} y_{i}$. Also $0=d \alpha\left(R, \frac{\partial}{\partial x_{i}}\right)=\sum_{i=1}^{n}\left(d x_{i} \wedge d y_{i}\right)\left(R, \frac{\partial}{\partial x_{i}}\right)$ gives $c_{i}=0$. Similarly, $0=d \alpha\left(R, \frac{\partial}{\partial y_{i}}\right)=\sum_{i=1}^{n}\left(d x_{i} \wedge\right.$ $\left.d y_{i}\right)\left(R, \frac{\partial}{\partial y_{i}}\right)$ gives $b_{i}=0$. Thus $a=1$ and $R=\frac{\partial}{\partial z}$.

(ii) Consider $\mathbb{R}^{3}$ with the contact form $\alpha=\sin (y) d x+\cos (y) d z$. Then the Reeb vector field is $R=\sin (y) \frac{\partial}{\partial x}+\cos (y) \frac{\partial}{\partial z}$.

Given two contact manifolds $(M, \alpha)$ and $\left(M^{\prime}, \alpha^{\prime}\right)$, let $h: M \longrightarrow M^{\prime}$ be a smooth map, that is, differentiable.

The map $h$ is called a morphism of contact manifolds, so long as $h^{*} \alpha^{\prime}=\alpha$ (Gatsé, 2016).

Given a contact diffeomorphism $h, h^{-1}$ is also contact, and $h$ is called a contactomorphism, where by definition of the pullback of a 1 -form $\alpha$

$$
\left(h^{*} \alpha\right)(x)(u)=\alpha(h(x))(d h(x) u)
$$

for $x$ element of $M$ and for any $u$ element of $T_{x} M$.

The main goal of this paper is to show that the contact manifolds are the Jacobi manifolds. We also will give the generalization of the notion of contactomorphism and will characterize the contact diffeomorphisms.

In what follows, $M$ denotes a paracompact and connected smooth manifold, $\mathscr{C}^{\infty}(M)$ the algebra of numerical functions of class $\mathscr{C}^{\infty}$ on $M, \mathscr{D}(M)$ the $\mathscr{C}^{\infty}(M)$-module of first order differential operators on $\mathscr{C}^{\infty}(M)$ and $\delta$ (Okassa, 2008) the cohomology operator associated with the identically map

$$
i d: \mathscr{D}(M) \longrightarrow \mathscr{D}(M) \text {. }
$$

The term "differential operator" will mean "first order differential operator".

\section{Link Between Jacobi Manifolds and Contact Manifolds}

Let $\Omega^{1}(M)_{R}$ be the $\mathscr{C}^{\infty}(M)$-module of differential forms of degree 1 on $M$ which vanish on $R$. Then the map

$$
\operatorname{ker} \alpha \longrightarrow \Omega^{1}(M)_{R}, X \longmapsto i_{X} d \alpha
$$

is an isomorphism of $\mathscr{C}^{\infty}(M)$-modules. Moreover, for any function $f \in \mathscr{C}^{\infty}(M)$, we obtain the following properties:

(i) $\theta_{f \cdot R} \alpha=d f$,

(ii) $\theta_{f \cdot R} d \alpha=0$, 
(iii) $\theta_{R}(f \alpha)=R(f) \cdot \alpha+d f$,

(iv) $\theta_{R} d(f \alpha)=(d R(f)) \wedge \alpha+R(f) \cdot d \alpha$.

The differential form $d f-R(f) \cdot \alpha$ vanishes on $R$ and we have easily

$$
[R, \operatorname{ker} \alpha] \subset \operatorname{ker} \alpha \text {. }
$$

One notes $X_{f}$ the unique vector field on $M$ belonging to $\operatorname{ker} \alpha$ such that

$$
i_{X_{f}} d \alpha=d f-R(f) \cdot \alpha .
$$

We notice that

(i) $R \in \operatorname{ker}\left(i_{X_{f}} d \alpha\right)$.

(ii) If $f=0$, then $X_{f}=0$.

(iii) If $f=1$, then $X_{f}=R$.

A differential operator on $M$ is a $\mathbb{R}$-linear map

$$
\varphi: \mathscr{C}^{\infty}(M) \longrightarrow \mathscr{C}^{\infty}(M)
$$

such that

$$
\varphi(f \cdot g)=\varphi(f) \cdot g+f \cdot \varphi(g)-f \cdot g \cdot \varphi(1)
$$

for any $f, g \in \mathscr{C}^{\infty}(M)$. We verify that the map

$$
\varphi_{f}=R(f)+X_{f}-f \cdot R: \mathscr{C}^{\infty}(M) \longrightarrow \mathscr{C}^{\infty}(M)
$$

is a differential operator on $M$. We set

$$
\{f, g\}=\varphi_{f}(g)
$$

we deduce the following results.

Theorem 3. For all $f, g$ and $h$ in $\mathscr{C}^{\infty}(M)$, we get
(i) $\left[X_{f}, X_{g}\right]=X_{\{f, g\}}+f \cdot X_{R(g)}-g \cdot X_{R(f)}+X_{f}(g) \cdot R$.
(ii) $\{f, g \cdot h\}=\{f, g\} \cdot h+g \cdot\{f, h\}-g \cdot h \cdot\{f, 1\}$.
(iii) $\left[R, \varphi_{f}\right]=\varphi_{R(f)}$.
(iv) $R\{f, g\}=\{R(f), g\}+\{f, R(g)\}$.
(v) $\left[\varphi_{f}, \varphi_{g}\right]=\varphi_{\{f, g\}}$.

Proof. (i) We check that

$$
i_{\left[X_{f}, X_{g}\right]-X_{\{f, g\}}-f \cdot X_{R(g)}+g \cdot X_{R(f)}-X_{f}(g) \cdot R} d \alpha=0 .
$$

(ii) The proof of this assertion is obvious.

(iii) For any $f \in \mathscr{C}^{\infty}(M)$, we get

$$
\begin{aligned}
{\left[R, \varphi_{f}\right] } & =\left[R, R(f)+X_{f}-f \cdot R\right] \\
& =[R, R(f)]+\left[R, X_{f}\right]-[R, f \cdot R] \\
& =R(R(f))+X_{R(f)}-R(f) \cdot R \\
& =\varphi_{R(f)} .
\end{aligned}
$$


(iv) Since

$$
R\left(X_{f}(g)\right)=X_{f}(R(g))+X_{g}(R(f))
$$

we deduce that

$$
R\{f, g\}=\{R(f), g\}+\{f, R(g)\} .
$$

(v) We calculate

$$
\begin{aligned}
{\left[\varphi_{f}, \varphi_{g}\right]=} & {\left[R(f)+X_{f}-f \cdot R, R(g)+X_{g}-g \cdot R\right] } \\
= & X_{f}(R(g))-f \cdot R(R(g))-X_{g}(R(f))+g \cdot R(R(f)) \\
& +\left[X_{f}, X_{g}\right]-X_{f}(g) \cdot R+g \cdot\left[R, X_{f}\right]+f \cdot\left[R, X_{g}\right] \\
& +X_{g}(f) \cdot R+(f \cdot R(g)-g \cdot R(f)) \cdot R \\
= & R\{f, g\}+X_{\{f, g\}}-\{f, g\} \cdot R \\
= & \varphi_{\{f, g\}}
\end{aligned}
$$

That ends the proof.

Proposition 4. The map

$$
\mathscr{C}^{\infty}(M) \times \mathscr{C}^{\infty}(M) \longrightarrow \mathscr{C}^{\infty}(M),(f, g) \longmapsto\{f, g\},
$$

defines a real Lie algebra structure on the contact manifold $M$.

Proof. We write

$$
\begin{aligned}
\{f,\{g, h\}\}+\{g,\{h, f\}\}+\{h,\{f, g\}\} & =\{f,\{g, h\}\}-\{g,\{f, h\}\}-\{\{f, g\}, h\} \\
& =\varphi_{f}\left[\varphi_{g}(h)\right]-\varphi_{g}\left[\varphi_{f}(h)\right]-\varphi_{\{f, g\}}(h) \\
& =\left(\left[\varphi_{f}, \varphi_{g}\right]-\varphi_{\{f, g\}}\right)(h) \\
& =0 .
\end{aligned}
$$

Hense the assertion is proved.

Give the foregoing, it is concluded that a contact manifold is a Jacobi manifold.

\section{Contactomorphism}

If $\mathscr{D}(M)$ is the set of differential operators on $M$ and

$$
\omega: \mathscr{D}(M) \times \mathscr{D}(M) \longrightarrow \mathscr{C}^{\infty}(M)
$$

is a nondegenerate skew-symmetric 2 -form on $M$, then the pair $(M, \omega)$ is a contact manifold (fore more details, see Okassa, 2011). Let

$$
i_{1} \omega: \mathscr{D}(M) \longrightarrow \mathscr{C}^{\infty}(M)
$$

be the 1-form on $M$ such that

$$
i_{1} \omega_{/ \mathscr{C}^{\infty}(M)}=0 .
$$

Proposition 5. (Okassa, 2008) If $\omega$ is a nondegenerate skew-symmetric 2-form such that $\delta \omega=0$, then $\omega=\delta\left(i_{1} \omega\right)$.

Proof. For all $\varphi$ and $\psi$ in $\mathscr{D}(M)$, we have

$$
\begin{aligned}
0 & =(\delta \omega)(1, \varphi, \psi) \\
& =\omega(\varphi, \psi)-\varphi\left[\left(i_{1} \omega\right)(\psi)\right]+\psi\left[\left(i_{1} \omega\right)(\varphi)\right]+\left(i_{1} \omega\right)[\varphi, \psi] \\
& =\left[\omega-\delta\left(i_{1} \omega\right)\right](\varphi, \psi)
\end{aligned}
$$

We deduce that $\omega=\delta\left(i_{1} \omega\right)$. 
Proposition 6. (Gatsé, 2019) Suppose that $f$ is a diffeomorphism on M. Let $\varphi$ be a differential operator of $\mathscr{C}^{\infty}(M)$. Then the map

$$
f_{*} \varphi=\left(f^{-1}\right)^{*} \circ \varphi \circ f^{*}: \mathscr{C}^{\infty}(N) \longrightarrow \mathscr{C}^{\infty}(N)
$$

is a differential operator of $\mathscr{C}^{\infty}(N)$.

Proposition 7. When $\varphi$ is a differential operator, when $f$ is a diffeomorphism then for any $g$ element of $\mathscr{C}^{\infty}(M)$, we obtain

$$
\varphi(g \circ f)=\left[\left(f_{*} \varphi\right)(g)\right] \circ f .
$$

Proof. Let $x$ be an element of $M$, we write

$$
\begin{aligned}
{[\varphi(g \circ f)](x) } & =\left[\varphi\left[f^{*}(g)\right]\right](x) \\
& =\left[f_{*} \varphi(g)\right][f(x)] \\
& =\left[\left[\left(f_{*} \varphi\right)(g)\right] \circ f\right](x) .
\end{aligned}
$$

Thus $\varphi(g \circ f)=\left[\left(f_{*} \varphi\right)(g)\right] \circ f$.

Proposition 8. If $f$ is a diffeomorphism and let $\xi$ be a multilinear $p$-form on $N$ then for all $\psi_{1}, \psi_{2}, \ldots, \psi_{p} \in \mathscr{D}(M)$,

$$
\left(f^{*} \xi\right)\left(\psi_{1}, \psi_{2}, \ldots, \psi_{p}\right)=\left[\xi\left(f_{*} \psi_{1}, f_{*} \psi_{2}, \ldots, f_{*} \psi_{p}\right)\right] \circ f .
$$

Proof. For $x$ element of $M$, we obtain

$$
\begin{aligned}
& \left(f^{*} \xi\right)\left(\psi_{1}, \psi_{2}, \ldots, \psi_{p}\right)(x) \\
= & \left(f^{*} \xi\right)(x)\left(\psi_{1}(x), \psi_{2}(x), \ldots, \psi_{p}(x)\right) \\
= & \xi[f(x)]\left[\left(f_{*} \psi_{1}, f_{*} \psi_{2}, \ldots, f_{*} \psi_{p}\right)(f(x))\right] \\
= & {\left[\xi\left(f_{*} \psi_{1}, f_{*} \psi_{2}, \ldots, f_{*} \psi_{p}\right)\right](f(x)) } \\
= & \left(\left[\xi\left(f_{*} \psi_{1}, f_{*} \psi_{2}, \ldots, f_{*} \psi_{p}\right)\right] \circ f\right)(x),
\end{aligned}
$$

then

$$
\left(f^{*} \xi\right)\left(\psi_{1}, \psi_{2}, \ldots, \psi_{p}\right)=\left[\xi\left(f_{*} \psi_{1}, f_{*} \psi_{2}, \ldots, f_{*} \psi_{p}\right)\right] \circ f
$$

That ends the proof.

Theorem 9. When $f$ is a diffeomorphism then $\delta$ and $f^{*}$ commute.

Proof. We have

$$
\begin{aligned}
& {\left[\delta\left(f^{*} \xi\right)\right]\left(\psi_{1}, \psi_{2}, \ldots, \psi_{p}, \psi_{p+1}\right) } \\
= & \sum_{i=1}^{p+1}(-1)^{i+1} \psi_{i}\left[\left(f^{*} \xi\right)\left(\psi_{1}, \psi_{2}, \ldots, \widehat{\psi}_{i}, \ldots, \psi_{p}, \psi_{p+1}\right)\right] \\
& +\sum_{i<j}(-1)^{i+j}\left(f^{*} \xi\right)\left(\left[\psi_{i}, \psi_{j}\right], \psi_{1}, \psi_{2}, \ldots, \widehat{\psi}_{i}, \ldots, \widehat{\psi}_{j}, \ldots, \psi_{p}, \psi_{p+1}\right) \\
= & {\left[\sum_{i=1}^{p+1}(-1)^{i+1}\left(f_{*} \psi_{i}\right)\left[\xi\left(f_{*} \psi_{1}, f_{*} \psi_{2}, \ldots, f_{*} \widehat{\psi}_{i}, \ldots, f_{*} \psi_{p+1}\right)\right]\right] \circ f } \\
& +\left[\sum_{i<j}(-1)^{i+j} \xi\left(\left[f_{*} \psi_{i}, f_{*} \psi_{j}\right], f_{*} \psi_{1}, \ldots, f_{*} \widehat{\psi}_{i}, \ldots, f_{*} \widehat{\psi}_{j}, \ldots, f_{*} \psi_{p}, f_{*} \psi_{p+1}\right)\right] \circ f .
\end{aligned}
$$

On the other hand, we get

$$
\begin{aligned}
& {\left[f^{*}(\delta \xi)\right]\left(\psi_{1}, \psi_{2}, \ldots, \psi_{p}, \psi_{p+1}\right) } \\
= & {\left[(\delta \xi)\left(f_{*} \psi_{1}, f_{*} \psi_{2}, \ldots, f_{*} \psi_{p+1}\right)\right] \circ f } \\
= & {\left[\sum_{i=1}^{p+1}(-1)^{i+1}\left(f_{*} \psi_{i}\right)\left[\xi\left(f_{*} \psi_{1}, f_{*} \psi_{2}, \ldots, f_{*} \widehat{\psi}_{i}, \ldots, f_{*} \psi_{p+1}\right)\right]\right] \circ f } \\
& +\left[\sum_{i<j}(-1)^{i+j} \xi\left(\left[f_{*} \psi_{i}, f_{*} \psi_{j}\right], f_{*} \psi_{1}, \ldots, f_{*} \widehat{\psi}_{i}, \ldots, f_{*} \widehat{\psi_{j}}, \ldots, f_{*} \psi_{p+1}\right)\right] \circ f .
\end{aligned}
$$


That ends the proof.

Proposition 10. If $f$ is a contactomorphism i.e. $f^{*} i_{1} \omega_{/ \mathfrak{X}(M)}=i_{1} \omega_{/ \mathfrak{X}(M)}$ then $f^{*} i_{1} \omega=i_{1} \omega$.

Proof. Suppose that $f$ is a contactomorphism then for any vector field $X$ on $M$, we obtain

$$
\left[\left(i_{1} \omega\right)\left(f_{*} X\right)\right] \circ f=\left(i_{1} \omega\right)(X) .
$$

For any $\varphi \in \mathscr{D}(M), \varphi=\varphi(1)+X$, and

$$
\begin{aligned}
\left(i_{1} \omega\right)\left(f_{*} \varphi\right) & =\left(i_{1} \omega\right)\left(f_{*} X\right) \\
& =\left[\left(i_{1} \omega\right)(X)\right] \circ f^{-1} \\
& =\left[\left(i_{1} \omega\right)(\varphi)\right] \circ f^{-1},
\end{aligned}
$$

then $\left[\left(i_{1} \omega\right)\left(f_{*} \varphi\right)\right] \circ f=\left(i_{1} \omega\right)(\varphi)$ so $f^{*} i_{1} \omega=i_{1} \omega$.

Theorem 11. The map $f$ is a contactomorphism if and only if $f^{*} \omega=\omega$.

Proof. Suppose that $f$ is a contactomorphism, for all $\varphi, \psi \in \mathscr{D}(M)$, we have

$$
\begin{aligned}
\omega\left(f_{*} \varphi, f_{*} \psi\right) & =\left[\delta\left(i_{1} \omega\right)\left(f_{*} \varphi, f_{*} \psi\right)\right] \\
& =\left(f_{*} \varphi\right)\left[\left(i_{1} \omega\right)\left(f_{*} \psi\right)\right]-\left(f_{*} \psi\right)\left[\left(i_{1} \omega\right)\left(f_{*} \varphi\right)\right]-\left(i_{1} \omega\right)\left[f_{*} \varphi, f_{*} \psi\right] \\
& =\varphi\left[\left(i_{1} \omega\right)(\psi)\right] \circ f^{-1}-\psi\left[\left(i_{1} \omega\right)(\varphi)\right] \circ f^{-1}-\left(i_{1} \omega\right)[\varphi, \psi] \circ f^{-1} \\
& =\omega(\varphi, \psi) \circ f^{-1}, \\
{\left[\omega\left(f_{*} \varphi, f_{*} \psi\right)\right] \circ f } & =\omega(\varphi, \psi) .
\end{aligned}
$$

Thus $f^{*} \omega=\omega$.

Conversely let $f^{*} \omega=\omega$. For $\varphi=1$, we have $f_{*} \varphi=1$. For $\varphi, \psi$ elements of $\mathscr{D}(M), \omega\left(f_{*} \varphi, f_{*} \psi\right)=\omega(\varphi, \psi) \circ f^{-1}$, then

$$
\begin{aligned}
\omega\left(1, f_{*} \psi\right) & =\omega(1, \psi) \circ f^{-1} \\
\left(i_{1} \omega\right)\left(f_{*} \psi\right) & =\left(i_{1} \omega\right)(\psi) \circ f^{-1} .
\end{aligned}
$$

In particular if $\psi=Y$, we have

$$
\left[\left(i_{1} \omega\right)\left(f_{*} Y\right)\right] \circ f=\left(i_{1} \omega\right)(Y)
$$

then $f^{*} i_{1} \omega_{/ \mathfrak{X}(M)}=i_{1} \omega_{/ \mathfrak{X}(M)}$.

That ends the proof.

Let $\mathscr{D}$ if $f_{\omega}(M)$ be the group of $\mathscr{C}^{\infty}(M)$-diffeomorphisms of $M$, which preserve $\omega$. This group is the automorphism group of the contact geometry of $(M, \omega)$. It acts transitively on the contact manifold (provided that is connected). Hence the contact manifold $(M, \omega)$ can be viewed as a homogeneous space of $\mathscr{D}$ if $f_{\omega}(M)$.

Theorem 12. Let $(M, \omega)$ and $\left(M^{\prime}, \omega^{\prime}\right)$ be two smooth contact manifolds equipped with contact structures $\omega$ and $\omega^{\prime}$ respectively. Let $\gamma: M \longrightarrow M^{\prime}$ be a bijective map such that for any map $f: M \longrightarrow M, \gamma f \gamma^{-1} \in \mathscr{D}$ iff $f_{\omega^{\prime}}\left(M^{\prime}\right)$ iff $f \in$ Dif $f_{\omega}(M)$. Then $\gamma$ is a $\mathscr{C}^{\infty}(M)$-diffeomorphism and $\gamma^{*} \omega^{\prime}=\omega$.

Proof. We use the Taken's theorem (see Takens, 1979) and the same arguments as in (Banyaga, 1986).

Theorem 13. Suppose a group isomorphism $\tau: \mathscr{D}$ if $f_{\omega}(M) \longrightarrow \mathscr{D}$ if $f_{\omega^{\prime}}\left(M^{\prime}\right)$ is also a homeomorphism when $\mathscr{D}$ if $f_{\omega}(M)$ and $\mathscr{D}$ iff $f_{\omega^{\prime}}\left(M^{\prime}\right)$ are endowed with the point-open topology. Then there exists a $\mathscr{C}^{\infty}(M)$-diffeomorphism $\gamma: M \longrightarrow M^{\prime}$ such that $\tau(f)=\gamma f \gamma^{-1}$, for any $f \in \mathscr{D}$ iff $f_{\omega}(M)$ and $\gamma^{*} \omega^{\prime}=\omega$.

Proof. Note that, these groups are $\gamma$-transitive. There exists a homeomorphism $\gamma: M \longrightarrow M^{\prime}$ such that $\tau(f)=\gamma f \gamma^{-1}$, for any $f \in \mathscr{D}$ if $f_{\omega}(M)$. Useing the above theorem, we have $\gamma^{*} \omega^{\prime}=\omega$.

Corollary 14. When an automorphism of the group of contact diffeomorphisms $\mathscr{D}$ iff $f_{\omega}(M)$ of contact manifold $(M, \omega)$ is also a homeomorphism for the point-open topology, then it is an inner automorphism. 


\section{A Characterization of Contact Diffeomorphisms}

Let

$$
\text { id }: \mathscr{D}(M) \longrightarrow \mathscr{D}(M)
$$

be the identically map of $\mathscr{D}(M)$. One notes $\delta=d_{i d}$, the cohomology operator associated with the representation $i d$. Let

$$
\omega: \mathscr{D}(M) \times \mathscr{D}(M) \longrightarrow \mathscr{C}^{\infty}(M)
$$

be a nondegenerate skew-symmetric 2 -form on $M$.

Proposition 15. When

$$
f: M \longrightarrow M
$$

satisfies $f^{*} \omega=\omega$, then for every function $u \in \mathscr{C}^{\infty}(M)$, we have

$$
f_{*} \varphi_{u}=\varphi_{u \circ f}
$$

\section{References}

Banyaga, A. (1986). On Isomorphic classical diffeomorphism group. I, Proc. Am. Math. Soc., 98(1), 113-118. https://doi.org/10.1090/S0002-9939-1986-0848887-5

Gatsé, S. C. ( 2016). Hamiltonian Vector Field on Locally Conformally Symplectic Manifold. International Mathematical Forum, 11(19), 933-941. http://dx.doi.org/10.12988/imf.2016.6666

Gatsé, S. C. ( 2019). Some Properties of first order differential operators, Advances in Pure Mathematics, 9(11), 934-943. https://doi.org/10.4236/apm.2019.911046

Koszul, J. L. (1985). Crochet de Schouten-Nijenhuis et cohomologie. Astérisque, 137(257-271), 4-3.

Lichnerowicz, A. (1977). Les variétés de Poisson et leurs algèbres de Lie associées. J. Differential Geometry, 12, 253-300. https://doi.org/10.4310/jdg/1214433987

Lichnerowicz, A. (1978). Les variétés de Jacobi et leurs algèbres de Lie associées. J. Math. pures et appl., 57(4), 453-488.

Okassa, E. (2007). Algèbres de Jacobi et algèbres de Lie-Rinehart-Jacobi. J. Pure Appl. Algebra, 208, 1071-1089. http://dx.doi.org/10.1016/j.jpaa.2006.05.013

Okassa, E. (2008). On Lie-Rinehart-Jacobi algebras. J. Algebra Appl., 7, 749-772. http://dx.doi.org/10.1142/s0219498808003107

Okassa, E. (2011). Symplectic Lie-Rinehart-Jacobi algebras and contact manifolds. Canad. Math. Bull., 54(4), 716-725. https://doi:10.4153/CMB-2011-033-6

Takens, F. (1979). Characterization of a differentiable structure by its group of diffeomorphisms. Bol. Soc. Brasil. Math., 10(1), 17-25. https://doi.org/10.1007/BF02588337

\section{Copyrights}

Copyright for this article is retained by the author(s), with first publication rights granted to the journal.

This is an open-access article distributed under the terms and conditions of the Creative Commons Attribution license (http://creativecommons.org/licenses/by/4.0/). 